\title{
Molecular-Genetic PET Imaging Using an HSV1-tk Mutant Reporter Gene with Enhanced Specificity to Acycloguanosine Nucleoside Analogs
}

Amer M. Najjar, Ryuichi Nishii, David S. Maxwell, Andrei Volgin, Uday Mukhopadhyay, William G. Bornmann William Tong, Mian Alauddin, and Juri G. Gelovani

Experimental Diagnostic Imaging, University of Texas M.D. Anderson Cancer Center, Houston, Texas

Imaging 2 different molecular-genetic events in a single subject by PET is essential in a variety of in vivo applications. Using herpes simplex virus-1 thymidine kinase (HSV1-tk) mutants with narrower substrate specificities in combination with wild-type HSV1-tk (wtHSV1-tk) would enable differential imaging with corresponding radiotracers, namely $2^{\prime}$-deoxy-2'-18 F-fluoro-5ethyl-1- $\beta$-D-arabinofuranosyl-uracil ( $\left.{ }^{18} \mathrm{~F}-\mathrm{FEAU}\right)$ and the acycloguanosine derivative 9-(4-18F-fluoro-3-[hydroxymethyl]butyl)guanine $\left({ }^{18} \mathrm{~F}-\mathrm{FHBG}\right)$. In this study, we evaluated wtHSV1-tk and the $\mathrm{A} 168 \mathrm{H}$ mutant, which has been reported to exhibit enhanced acycloguanosine substrate catalytic activity and diminished pyrimidine phosphorylating activity, as PET reporter genes. Methods: Computational analysis was performed to assess the binding mode of FHBG and FEAU to wtHSV1-tk and the A168H variant. U87 cells were stably transduced with wtHSV1-tk or HSV1-tk(A168H) fused with green fluorescent protein and sorted to obtain equivalent transgene expression. In vitro uptake studies were performed to determine rates of substrate accumulation and retention. Nude mice bearing tumors expressing HSV1-tk variants were subsequently imaged using ${ }^{18} \mathrm{~F}-\mathrm{FHBG}$ and ${ }^{18} \mathrm{~F}-\mathrm{FEAU}$. Results: Docking results indicate that binding of $F H B G$ to the $A 168 \mathrm{H}$ variant is unaffected whereas the binding of FEAU is hindered because of a steric clash with the bulkier mutant residues. U87 cells expressing HSV1-tk(A168H) accumulated ${ }^{18} \mathrm{~F}-\mathrm{FHBG}$ in in vitro uptake studies at a 3 -fold higher rate than did cells expressing wtHSV1-tk without any detectable accumulation of ${ }^{3} \mathrm{H}-\mathrm{FEAU}$. Furthermore, HSV1-tk(A168H) demonstrated no thymidine phosphorylation activity. In contrast, U87 cells expressing wtHSV1-tk preferentially accumulated ${ }^{3} \mathrm{H}-$ FEAU at an 18 -fold higher rate than they did ${ }^{18} \mathrm{~F}-\mathrm{FHBG}$. Tumors expressing wtHSV1-tk or HSV1-tk $(\mathrm{A} 168 \mathrm{H})$ were distinctly imaged with ${ }^{18} \mathrm{~F}-\mathrm{FEAU}$ or ${ }^{18} \mathrm{~F}-\mathrm{FHBG}$, respectively. Hence, tumors expressing HSV1-tk $(\mathrm{A} 168 \mathrm{H})$ accumulated 8.4-fold more ${ }^{18} \mathrm{~F}$ FHBG than did tumors expressing wtHSV1-tk. In addition, wtHSV1-tk tumors, compared with HSV1-tk(A168H)-expressing tumors (which retained baseline levels of the radiotracer), preferentially accumulated ${ }^{18} \mathrm{~F}-\mathrm{FEAU}$. Conclusion: The FEAU and FHBG substrate discrimination capacity of the wtHSV1-tk and

Received Mar. 18, 2008; revision accepted Nov. 24, 2008.

For correspondence or reprints contact: Juri G. Gelovani, Experimental Diagnostic Imaging, University of Texas M.D. Anderson Cancer Center,

Houston, TX 77030.

E-mail: jgelovani@di.mdacc.tmc.edu

COPYRIGHT @ 2009 by the Society of Nuclear Medicine, Inc.
HSV1-tk(A168H) reporter enzymes was validated in vivo by PET of mice with tumor xenografts established from U87 cells expressing these different reporters. Thus, HSV1-tk(A168H) may potentially be used as a second reporter gene in combination with wtHSV1-tk to achieve differential PET.

Key Words: HSV1-tk; A168H; ${ }^{18}$ F-FHBG; ${ }^{18}$ F-FEAU

J Nucl Med 2009; 50:409-416

DOI: 10.2967/jnumed.108.058735

$\mathbf{P}$ modality. In combination with various reporter geneimaging approaches, PET allows for monitoring different cellular processes such as transcriptional regulation, signal transduction, protein-protein interactions, oncogenic transformation, cell trafficking, and targeted drug action. This monitoring is achieved through the incorporation of reporter genes and use of corresponding reporter probes (radiotracers) labeled with positron-emitting radionuclides. Reporter gene-mediated binding, accumulation, and retention of these positron-emitting reporter probes in target cells enable repetitive spatial-temporal dynamic imaging of molecular and cellular events by PET (1-3).

The concept of noninvasive reporter gene imaging with nuclear imaging modalities ( $\gamma$-camera, SPECT, PET) was introduced for the first time by Tjuvajev et al. and involved herpes simplex virus-1 thymidine kinase (HSV1-tk) as a model reporter gene (4). HSV1-tk phosphorylates a broad range of nucleoside analogs, and its expression can be imaged with several substrate analogs including $5-{ }^{124 / 131} \mathrm{I}-$ 2 -deoxy-2' -fluoro-1- $\beta$-D-arabino-uracil ( ${ }^{124 / 131}$ I-FIAU) $(4,5)$, $2^{\prime}$-deoxy-2' - ${ }^{18}$ F-fluoro-5-ethyl-1- $\beta$-D-arabinofuranosyl-uracil $\left({ }^{18} \mathrm{~F}-\mathrm{FEAU}\right)(6-8)$, or acycloguanosine derivatives, such as 9-(4- ${ }^{18}$ F-fluoro-3-[hydroxymethyl]butyl)guanine ( $\left.{ }^{18} \mathrm{~F}-\mathrm{FHBG}\right)$ (9-11). These nucleoside analogs are not readily phosphorylated by mammalian thymidine kinases but are selectively phosphorylated by HSV1-tk to their monophosphate forms and accumulate in HSV1-tk-expressing cells. Subsequent conver- 
sion of the monophosphorylated nucleosides into di- and triphosphates further enhances the trapping of the radiolabeled probes. The location and magnitude of radioactivity accumulation can be imaged noninvasively and repetitively in 3 dimensions using conventional preclinical and clinical PET instruments $(5,12,13)$.

The broad substrate specificity of HSV1-tk provides an array of imaging radiotracers, each enabling monitoring of single molecular-genetic or signal transduction processes in vivo $(1,10,13,14)$. However, with the rapidly developing field of systems biology, the molecular imaging field is moving toward the visualization and quantitation of multiple processes in the same or different target cell populations. Therefore, additional reporter genes that can be used in parallel with HSV1-tk are required to visualize temporal dynamics and spatial heterogeneity of multiple molecular events in target tissues.

Several PET techniques using HSV1-tk in combination with other PET reporter genes have been previously reported. The spatial heterogeneity and dynamics of hypoxia-inducible factor-1 $\alpha$ (HIF-1 $\alpha)$ expression in tumor xenografts, for example, has been described using HIF-1 $\alpha$ promoter-driven expression of HSV1-tk in combination with a constitutively expressed xanthine phosphoribosyltransferase beacon reporter gene to monitor cell viability (6). Human mitochondrial thymidine kinase 2 (hTK2) may also be potentially used as a secondary reporter gene because it exhibits narrower substrate specificity than does HSV1-tk (15). Kummer et al. have previously described a method of multitracer imaging using a bicistronic gene cassette expressing a mutant dopamine type 2 receptor (d2r80A) and HSV1-tk. Colocalized signals were obtained using the ${ }^{11} \mathrm{C}$-labeled $\mathrm{d} 2 \mathrm{r} 80 \mathrm{~A}$ ligand raclopride and ${ }^{18}$ F-FHBG (16). Another imaging approach uses the sodium/iodide symporter as a reporter gene in combination with HSV1-tk (17). The d2r80A and sodium/ iodide symporter imaging systems can potentially be coupled with hTk 2 to achieve differential imaging of 2 or 3 reporter genes.

These modalities, however, possess inherent limitations that may hinder their practical application in differential imaging. Although hTK2 does not catalyze ${ }^{18} \mathrm{~F}-\mathrm{FHBG}$, it exhibits a shared catalytic activity for FEAU and ${ }^{124 / 131} \mathrm{I}-$ FIAU precluding its use with HSV1-tk for differential imaging purposes (15). Compared with enzymatic reporters, which are not saturable at tracer levels of substrate and result in signal amplification, receptor-based reporters such as d2r80A are potentially saturable, especially at low levels of expression, limiting radiotracer accumulation and PET signal intensity.

Furthermore, pairing of fundamentally different PET reporter genes (i.e., enzymes with receptors or transporters) may introduce extraneous experimental variables and uncertainties. Thus, a more adequate and comparable assessment of distinct events may be achieved using variants of similar reporter genes. For example, an ideal 2-reporter imaging system should use 2 structurally related reporters (i.e., mutants derived from the same gene), which would likely have similar subcellular localization, protein halflife, and reporter enzyme-transporter-receptor densities. Similarly, it would be preferable to use 2 separate probes with similar structures and acid dissociation constant values and hence comparable pharmacokinetics and biologic distributions.

We sought to identify genetic mutants of the HSV1-tk enzyme possessing narrower substrate specificities. Ideally, these HSV1-tk mutants should possess unique substrate specificities without overlapping catalytic activities. Recently, Balzarini et al. have reported results of a comprehensive study of HSV1-tk variants containing amino acid substitutions at positions 167 or 168 (18). One of these variants, HSV1tk $(\mathrm{A} 168 \mathrm{H})$ - compared with wtHSV1-tk- has preserved purine and diminished pyrimidine phosphorylation activities. Hence, the thymidine (Thd) catalytic rate constant $\left(\mathrm{K}_{\text {cat }}\right)$ values for HSV1-tk(A168H) are drastically reduced by several orders of magnitude, which would potentially eliminate Thd as an endogenous competitor for radiolabeled PET probes. Furthermore, bromovinyl-deoxyuridine, a pyrimidine, does not inhibit phosphorylation of ganciclovir by HSV1-tk $(\mathrm{A} 168 \mathrm{H})$, suggesting that the mutant would also not phosphorylate FEAU given its structural similarity to bromovinyldeoxyuridine. The presence of any C5 group larger than a methyl on the pyrimidine nucleosides would also clash with A168H and hinder effective docking into the active site.

HSV1-tk(A168H) also demonstrates an increased catalytic activity for acycloguanosine substrates, exhibiting a 4.3-fold increase in $\mathrm{K}_{\mathrm{cat}}$ for ganciclovir over wtHSV1-tk. On the basis of these observations, the A168H mutant of HSV1-tk would be expected to discriminate against FEAU while phosphorylating the acycloguanosine ${ }^{18} \mathrm{~F}-\mathrm{FHBG}$ at a higher catalytic rate than wtHSV1-tk. We hypothesized, therefore, that the enhanced specificities exhibited by these variants may be adequate for achieving differential PET reporter imaging capabilities.

In this study, the feasibility of using wtHSV1-tk in combination with HSV1-tk(A168H) for the purpose of differential PET was evaluated by molecular modeling studies to determine the structure-activity relationships of FEAU and FHBG in binding to wtHSV1-tk and the mutant enzyme, analysis of the rate of radiotracer accumulation in tumor cells expressing the enzymes, and in vivo imaging of animals bearing tumor xenografts expressing wild-type and mutant HSV1-tk with ${ }^{18} \mathrm{~F}-\mathrm{FEAU}$ and ${ }^{18} \mathrm{~F}-\mathrm{FHBG}$, respectively.

\section{MATERIALS AND METHODS}

\section{In Silico Evaluation of Acycloguanosine Substrate Docking}

Preparation of Structures for Docking. The radiographic crystal structures of HSV1-tk with Thd (code 1KIM) and ganciclovir (code 1KI2) were obtained from the Protein Data Bank (19). The structures were loaded into Sybyl 7.2 (Tripos, Inc.) and prepared for assisted model building and energy refinement with AMBER 
8 (20). The method consisted of removing the ligand and all waters, adding hydrogens, and creating the $\mathrm{A} 168 \mathrm{H}$ mutant using the BioPolymer menu in Sybyl. Torsions for the mutants were manually corrected to avoid steric clashes with nearby residues. Pyrimidine and purine substrates were derived through modification of the existing Thd and ganciclovir structures. A Concord (Robert S. Pearlman, University of Texas, Austin, distributed by Tripos Inc.) clean-up was used to generate the initial conformation for docking. Structures from AMBER were prepared for docking by adding back the respective ligand. Docking was completed with the Surflex $(21,22)$ as distributed in Sybyl 7.3. The protocol was generated using the ligand mode and crystal substrate, with default settings of 0.50 for the threshold and 0 for bloat. Three additional starting configurations of the substrate were selected to avoid bias of starting configuration. The side chain of N125 is oriented differently with respect to the purine or pyrimidine ligand, and because receptor flexibility is not considered during Surflex docking, it was necessary to use separate structures. Therefore, 10 final docked configurations were requested for wtHSV1-tk and 2 mutants on the basis of both the 1KIM and $1 \mathrm{KI} 2$ structures.

\section{Site-Directed Mutagenesis}

Site-directed mutagenesis was performed on an HSV1-tk/green fluorescent protein (GFP) fusion gene cloned into an SFG-based retroviral backbone (SFG-nesHSVTK/GFP) (23). A QuikChange mutagenesis kit (Stratagene) was used to generate base-substitution mutations using 3 forward and reverse primer pairs designed to target A168 of HSV1-tk. The forward primer 5'-cttcgaccgccatcccatcgcccacctcetgtgctacceggcc- $3^{\prime}$ was used with its corresponding complementary primer to produce the HSV1-tk mutants.

\section{Stable Retroviral Transduction of U87 Cells}

The vesicular stomatitis virus G-pseudotyped retrovirus was produced by transfecting 293GPG packaging cells (kindly provided by Daniel S. Ory) with HSV1-tk constructs using Lipofectamine 2000 (Invitrogen) in 6-well plates according to the manufacturer's protocol (24). The transfected cells were then expanded into 100$\mathrm{mm}$ dishes, and fresh medium without tetracycline was added $48 \mathrm{~h}$ later to induce vesicular stomatitis virus $\mathrm{G}$ expression and virus packaging. Two 3-dimensional supernatants were collected, filtered through low-protein binding filters $(0.22 \mu \mathrm{m})$, and stored at $-80^{\circ} \mathrm{C}$. U87 cells were plated in 6-well plates at a density of $2 \times$ $10^{5} / \mathrm{mm}^{2}$ and incubated for $24 \mathrm{~h}$. A $1: 1$ mixture of retroviral supernatant and Dulbecco's modified Eagle's medium/F12 medium (Invitrogen) supplemented with $10 \%$ fetal bovine serum was added to the monolayers in the presence of $6 \mu \mathrm{g}$ of polybrene per milliliter (Sigma-Aldrich). After a 48-h incubation, fresh medium was added, and the cells were incubated for an additional $48 \mathrm{~h}$. The monolayers were then expanded into 100-mm dishes. Expression of the HSV1-tk/GFP fusion gene was assessed by fluorescenceactivated cell sorting (FACS) and reverse-transcriptase polymerase chain reaction $(\mathrm{PCR})$ analysis.

\section{FACS and Gene Expression Analysis}

Transduced cells were analyzed by FACS to assess and equate the expression of the HSV1-tk/GFP fusion gene in all transduced cell lines. FACS analysis was performed on a FACSCalibur (BD Biosciences) using a 488-nm excitation laser. To equate HSV1-tk/ GFP expression levels in all cell lines, a cell-sorting instrument (FACSAria; BD Biosciences) was used to collect cells within a narrow region spanning $10^{2}-10^{3}$ on the GFP expression scale of a histogram plot. Sorted cells were then reanalyzed by FACS. HSV1-tk/GFP expression was also measured by quantitative PCR using an ABI 7500 instrument (Applied Biosystems). The following primer pairs were used to detect HSV1-tk and the endogenous standard glyceraldehyde-3-phosphate dehydrogenase (GAPDH): 5' -TAACAATGGGCATGCCTTATGCCG-3' (HSVTK-F), 5'-ACCGTATTGGCAAGTAGCCCGTAA-3' (HSVTK-R), 5' CCATGGAGAAGGCTGGGG-3' (GAPDH-F), 5' -CAAAGTTGTCATGGATGACC-3' (GAPDH-R). Expression of HSV1-tk/GFP was normalized to GAPDH, and results were expressed as change in $\mathrm{C}_{\mathrm{t}}\left(\Delta \mathrm{C}_{\mathrm{t}}\right)$ values.

\section{In Vitro Uptake Study}

Transduced U87 cells expressing individual HSV1-tk mutants were plated in $150-\mathrm{mm}$ dishes in triplicate at subconfluent densities and incubated for $24 \mathrm{~h}$. The medium was replaced with $14 \mathrm{~mL}$ of medium containing radiotracers ${ }^{14} \mathrm{C}$-Thd $(0.37 \mathrm{kBq} / \mathrm{mL})$ (Moravek), ${ }^{3} \mathrm{H}-\mathrm{FEAU}$ (3.7 kBq/mL) (Moravek), and ${ }^{18} \mathrm{~F}-\mathrm{FHBG}$ $(92.5 \mathrm{kBq} / \mathrm{mL}$ at time 0$)$. The cells were subsequently incubated for $15,30,60$, and 120 min before the monolayers were scraped, transferred into $15-\mathrm{mL}$ tubes, and centrifuged at $1,000 \mathrm{~g}$ for $2 \mathrm{~min}$. A $100-\mu \mathrm{L}$ aliquot of supernatant was transferred to a preweighed scintillation tube, and the rest was removed by aspiration before the cell pellet was snap-frozen on dry ice. The frozen pellets were transferred to preweighed scintillation vials, weighed, and thoroughly resuspended in $0.5 \mathrm{~mL}$ of Soluene-350 (Perkin Elmer). Radioactive $\gamma$-emissions of the medium and the cell pellets were first measured on a $\gamma$-counter (Cobra Quantum; Packard) to quantify ${ }^{18} \mathrm{~F}$-FHBG uptake. After the decay of ${ }^{18} \mathrm{~F}$ activity, $\beta$-emissions were measured within precalibrated energy ranges using a scintillation counter (Tri-Carb 3100TR; Packard) to quantify ${ }^{3} \mathrm{H}-\mathrm{FEAU}$ and ${ }^{14} \mathrm{C}$-Thd uptakes. Activity ratios of the cell pellet to medium ([dpm/g cells $] /[\mathrm{dpm} / \mathrm{g}$ medium $]$ ) were calculated and plotted against time.

\section{Radiotracers}

${ }^{18} \mathrm{~F}-\mathrm{FHBG}$ and ${ }^{18} \mathrm{~F}-\mathrm{FEAU}$ were synthesized following literature methods developed in our laboratory, with high purity and high specific activity as described (9).

\section{Animal Model}

Animal studies were performed under an approved Institutional Animal Care and Use Committee protocol. Tumors were grown in 4-wk-old athymic nude mice ( $n=6$, Charles River Laboratories). Two tumor types were injected contralaterally in the dorsal shoulder region of each animal $\left(1 \times 10^{6}\right.$ cells $/ 100 \mu \mathrm{L}$ Matrigel [BD Biosciences]): U87 wtHSV1-tk (left) and U87 HSV1-tk(A186H) (right). Approximately $10 \mathrm{~d}$ later, when tumors were about $1 \mathrm{~cm}$ in diameter, small-animal PET was performed.

\section{Small-Animal PET Imaging Studies}

Studies were performed using a microPET-R4 (Concorde Microsystems). Tumor-bearing animals were anesthetized using isoflurane $(2.0 \%)$ in $98 \%$ oxygen and placed in a prone position under a heat lamp for a dynamic scan. The radiotracer ${ }^{18} \mathrm{~F}-\mathrm{FHBG}$ (3.7 $\mathrm{MBq}$ in $100 \mu \mathrm{L}$ of saline) was administered via the tail vein, and 2-dimensional-mode dynamic PET scans were acquired for $120 \mathrm{~min}$. The following day, the same group of animals was injected with ${ }^{18} \mathrm{~F}-\mathrm{FEAU}$ (3.7 $\mathrm{MBq}$ in $100 \mu \mathrm{L}$ of saline), and dynamic PET scans were acquired for $120 \mathrm{~min}$. On the third day, 
small-animal PET imaging with ${ }^{18}$ F-FDG (3.7 MBq in $100 \mu \mathrm{L}$ of saline) (PETNET) was performed, and static scans were obtained $1 \mathrm{~h}$ after injection of the radiotracer. The radiotracer uptake levels in the tumor, blood (aorta), and muscle were determined and expressed as percentage injected dose per gram $(\% \mathrm{ID} / \mathrm{g}) \pm \mathrm{SD}$.

\section{RESULTS}

\section{Computational Analysis Predicts Altered Substrate}

Binding Specificity of HSV1-tk (A168H)

The binding mode of FEAU and ${ }^{18} \mathrm{~F}-\mathrm{FHBG}$ to the A168H variant was hypothesized to be consistent with Thd and ganciclovir binding, as previously reported by Balzarini et al. (18). Thus, computational analysis was performed to predict the binding mode of the FEAU and ${ }^{18} \mathrm{~F}-\mathrm{FHBG}$ to wtHSV1-tk and the A168H variant. The docking results are based on the overall score and match to the crystal binding mode. Pyrimidine and purine substrates were docked into the respective Protein Data Bank crystal structures determined with Thd (1KIM) and ganciclovir (1KI2) ligands, in wild-type and mutant forms, to maintain conformational consistency. The pyrimidine substrates docked with sufficiently high scores (average, 6.29) and correct binding mode only to the wtHSV1-tk form. In contrast, the docking scores of the pyrimidine substrate for the A168H mutant were significantly lower, yielding an average 2.09. Purine substrate (FHBG, ganciclovir, penciclovir) docking produced consistently high scores for wtHSV1-tk and HSV1-tk(A168H), yielding average values of 8.36 and 8.98 , respectively. The binding conformation of FHBG in wtHSV1-tk and in the mutant form is shown in Figure 1. The primary binding of FHBG is apparently not affected by the A168H mutation. The steric clash between the C5 methyl group of the pyrimidine nucleosides and H168 appears to be the primary factor, preventing proper binding of FEAU to the mutated active sites as illustrated in Figure 1B.
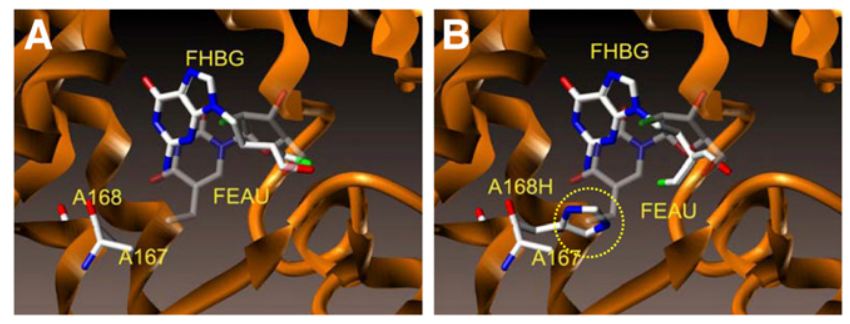

FIGURE 1. A168H mutation selectively hinders FEAU binding but does not hinder ${ }^{18} \mathrm{~F}-\mathrm{FHBG}$ binding. Computational analysis predicted reduced affinity of $\mathrm{A} 168 \mathrm{H}$ mutant of HSV1-tk for purine FEAU, whereas binding capacity of pyrimidine ${ }^{18} \mathrm{~F}-\mathrm{FHBG}$ is preserved. Spatial configuration within active site of wtHSV1-tk permits binding of both FEAU and ${ }^{18} \mathrm{~F}-\mathrm{FHBG}(\mathrm{A})$. A168H mutation (B) alters spatial configuration of active site, leading to steric clash between bulkier side chains and C5 ethyl group of FEAU (circled).
Transduced Cell Lines Express Equivalent Levels of HSV1-tk/GFP

To assess and compare FEAU and FHBG radiotracer uptake in vitro and to evaluate differential PET capacities of the HSV1-tk variants, retrovirally transduced U87 cells expressing HSV1-tk/GFP fusion proteins (wild-type and A168H) were produced. To yield cells expressing equivalent levels of transgene for accurate comparative analysis of the HSV1-tk variants in vivo and in vitro, transduced cell lines were uniformly sorted by FACS. Subsequent analysis of the sorted cell lines demonstrated an equivalent gaussian distribution of HSV1-tk/GFP expression in all transduced cell lines (Fig. 2). Equivalent transgene expression was further confirmed by quantitative PCR analysis, in which the presort $\Delta \mathrm{C}_{\mathrm{t}}$ values for U87 wtHSV1-tk and HSV1tk $(\mathrm{A} 168 \mathrm{H})$ were 4.62 and 5.01, respectively. Subsequent sorting resulted in more uniform $\Delta C_{t}$ values of 4.92 and 4.94 .

\section{Wild-Type HSV1-tk and HSV1-tk A186H Exhibit Inverse} Substrate Specificities for PET Radiotracers

Computational analysis indicated that mutant forms of HSV1-tk retain their ability to bind FHBG in the correct binding mode while discriminating against the pyrimidine nucleoside FEAU. In vitro radiotracer uptake studies were performed to determine the catalytic specificity of the HSV1tk variants by measuring the rate of intracellular accumulation and retention of the radiotracers. The accumulation of ${ }^{14} \mathrm{C}-\mathrm{Thd},{ }^{3} \mathrm{H}-\mathrm{FEAU}$, and ${ }^{18} \mathrm{~F}-\mathrm{FHBG}$ was studied simultaneously (triple-label study) in untransduced and transduced U87 cells stably expressing wtHSV1-tk and HSV1tk(A168H) (Fig. 3). As expected, untransduced U87 cells did not exhibit any detectable uptake and accumulation of ${ }^{3} \mathrm{H}-\mathrm{FEAU}$ or ${ }^{18} \mathrm{~F}-\mathrm{FHBG} .{ }^{14} \mathrm{C}$-Thd uptake at a rate of 0.0980 $\min ^{-1}$ was attributed to endogenous thymidine kinase 1 activity and was established as a baseline value for Thd uptake

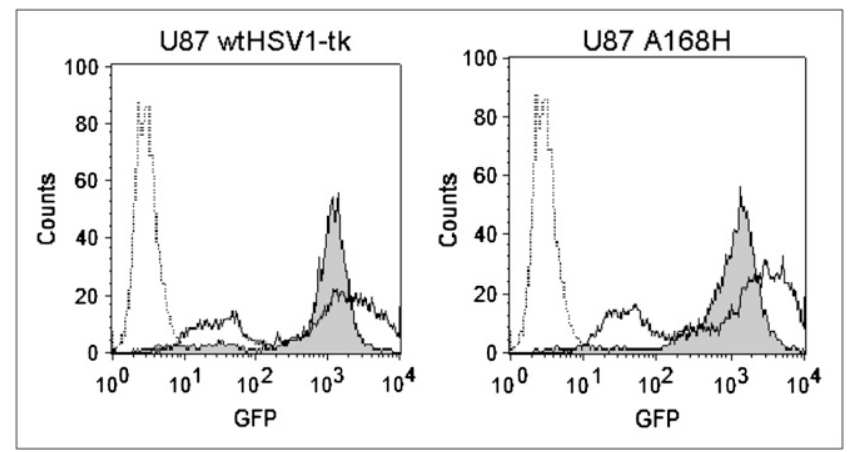

FIGURE 2. U87 cells transduced with HSV1-tk-GFP express equivalent levels of transgene after sorting. U87 HSV1-tk/GFP-expressing cell lines were sorted uniformly based on GFP. FACS analysis of unsorted cell lines yielded wide distribution of fluorescence intensities (solid line). Sorting produced uniform gaussian distributions of fluorescence intensities in all cell lines (shaded area). Untransduced cell line profile is also shown (dotted line). 


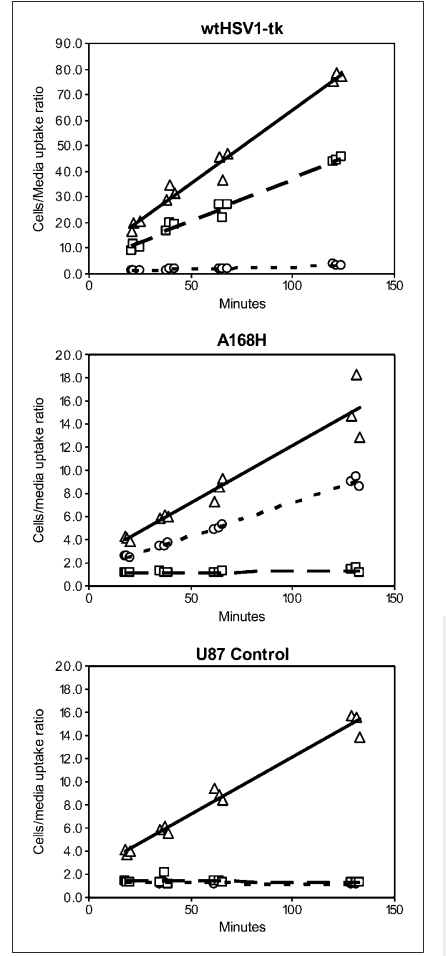

FIGURE 3. HSV1-tk variants exhibit differential uptake of FEAU and ${ }^{18} \mathrm{~F}-$ FHBG in vitro. Uptake and accumulation of ${ }^{14} \mathrm{C}$-Thd $(\triangle),{ }^{3} \mathrm{H}-\mathrm{FEAU}(\square)$, and ${ }^{18} \mathrm{~F}-$ FHBG $(\bigcirc)$ were determined in HSV1-tk-expressing U87 cells.

in the U87 cells. The cells expressing wtHSV1-tk demonstrated preferential uptake of ${ }^{3} \mathrm{H}$-FEAU over ${ }^{18} \mathrm{~F}-\mathrm{FHBG}$ (at rates of $0.335 \pm 0.015$ and $0.019 \pm 0.002 \mathrm{~min}^{-1}$, respectively) and a ${ }^{14} \mathrm{C}$-Thd uptake rate of $0.570 \pm 0.026 \mathrm{~min}^{-1}$, 5.8-fold higher than the baseline level, indicating Thd phosphorylation activity by wtHSV1-tk. In contrast, HSV1tk $(\mathrm{A} 168 \mathrm{H})$ yielded inverse specificities, exhibiting rates of less than $0.001 \mathrm{~min}^{-1}$ for ${ }^{3} \mathrm{H}-\mathrm{FEAU}$ and $0.058 \pm 0.002$ $\mathrm{min}^{-1}$ for ${ }^{18} \mathrm{~F}$-FHBG. Moreover, the rate of ${ }^{14} \mathrm{C}$-Thd uptake was similar to the baseline level established in the control, indicating a diminished capacity to phosphorylate pyrimidine nucleosides.
${ }^{18}$ F-FHBG and ${ }^{18}$ F-FEAU Can Be Used to Differentially Image Xenografts Expressing wtHSV1-tk or HSV1-tk(A168H)

The contrasting substrate specificities exhibited by wtHSV1-tk and the A168H mutant in the in vitro uptake studies indicated that the enzymes could be used for differential imaging studies in which ${ }^{18} \mathrm{~F}-\mathrm{FEAU}$ and ${ }^{18} \mathrm{~F}-$ FHBG can be used to selectively monitor wtHSV1-tk- and HSV1-tk(A168H)-expressing tumors in the same animal. U87 tumors expressing these HSV1-tk variants were used to validate the feasibility and efficacy of differential PET (Fig. 4). PET with ${ }^{18}$ F-FDG demonstrated similar viability and glycolytic activity in tumor xenografts, which was manifested by equivalent accumulation of the radiotracer in wtHSV1-tk U87 tumors $(\% \mathrm{ID} / \mathrm{g}=4.09 \pm 0.50)$ and HSV1-tk(A168H)-expressing tumors $(\% \mathrm{ID} / \mathrm{g}=4.52 \pm$ 0.69). Specific accumulation of ${ }^{18} \mathrm{~F}-\mathrm{FEAU}$ was evident in wtHSV1-tk tumors, whereas no ${ }^{18} \mathrm{~F}-\mathrm{FEAU}$ accumulation above background tissue levels was detected in the HSV1$\mathrm{tk}(\mathrm{A} 168 \mathrm{H})-$ expressing tumors. Accumulation of ${ }^{18} \mathrm{~F}-\mathrm{FHBG}$ was observed in HSV1-tk(A168H)-expressing tumors, compared with wtHSV1-tk-expressing tumors, in which no significant accumulation of the radiotracer was detected.

Figure 5 illustrates the time-activity curve of ${ }^{18} \mathrm{~F}-\mathrm{FHBG}$ and ${ }^{18} \mathrm{~F}-\mathrm{FEAU}$ in the blood, muscle, and tumors expressing wtHSV1-tk and the HSV1-tk(A168H) mutant. Maximum resolution of radiotracer accumulation in the tumors was observed at $120 \mathrm{~min}$. The $\% \mathrm{ID} / \mathrm{g}$ of ${ }^{18} \mathrm{~F}$-FEAU in U87 tumors expressing wtHSV1-tk was $4.6 \pm 0.17$. In contrast, the $\% \mathrm{ID} / \mathrm{g}$ value in HSV1-tk(A168H)-expressing tumors was of $2.2 \pm 0.22$, equivalent to the blood and muscle values of $1.96 \pm 0.15$ and $1.95 \pm 0.23$, respectively. U87 tumors expressing HSV1-tk(A168H) exhibited preferential (8.4-fold) accumulation of ${ }^{18} \mathrm{~F}-\mathrm{FHBG}(\% \mathrm{ID} / \mathrm{g}=1.77 \pm$ 0.53 ), compared with wtHSV1-tk-expressing tumors (\%ID/ $\mathrm{g}=0.21 \pm 0.11)$, blood $(\% \mathrm{ID} / \mathrm{g}=0.14 \pm 0.02)$, and muscle $(\% \mathrm{ID} / \mathrm{g}=0.15 \pm 0.07)$.

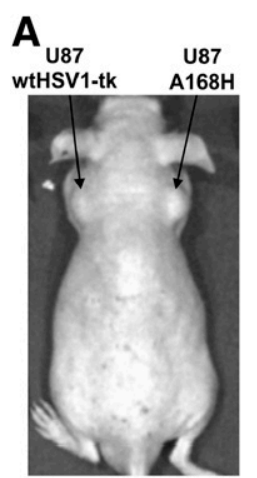

B
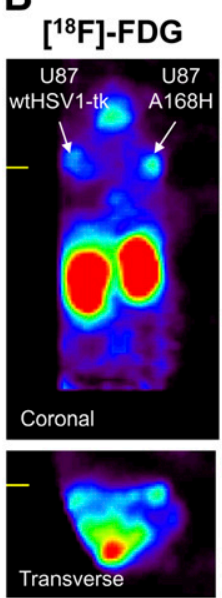

C

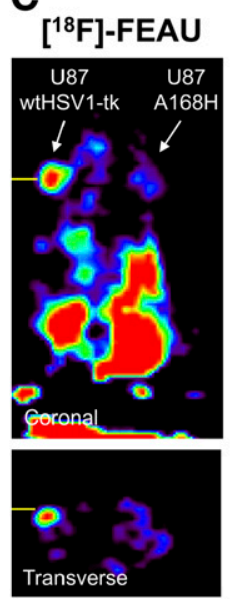

D

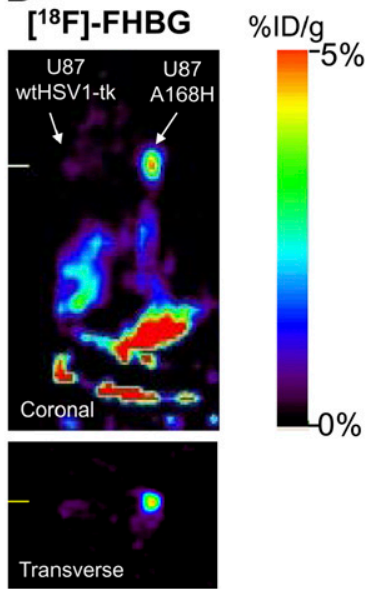

FIGURE 4. U87 cells expressing wtHSV1-tk and HSV1-tk(A168H) can be differentially imaged with ${ }^{18} \mathrm{~F}-\mathrm{FHBG}$ and ${ }^{18} \mathrm{~F}-\mathrm{FEAU}$. U87 wtHSV1-tk and U87 HSV1-tk(A168H) tumors were injected contralaterally in athymic nude mice (A). Both tumors were visible with equivalent intensities when imaged with ${ }^{18} \mathrm{~F}-\mathrm{FDG}$ $1 \mathrm{~h}$ after injection (B). Imaging with ${ }^{3} \mathrm{H}-$ FEAU exclusively revealed U87 wtHSV1tk tumor $2 \mathrm{~h}$ after injection of radiotracer (C), and U87 HSV1-tk(A168H)-expressing tumor was resolved using ${ }^{18} \mathrm{~F}-\mathrm{FHBG}$ (D). Signal intensities for all images are displayed using standardized scale. 


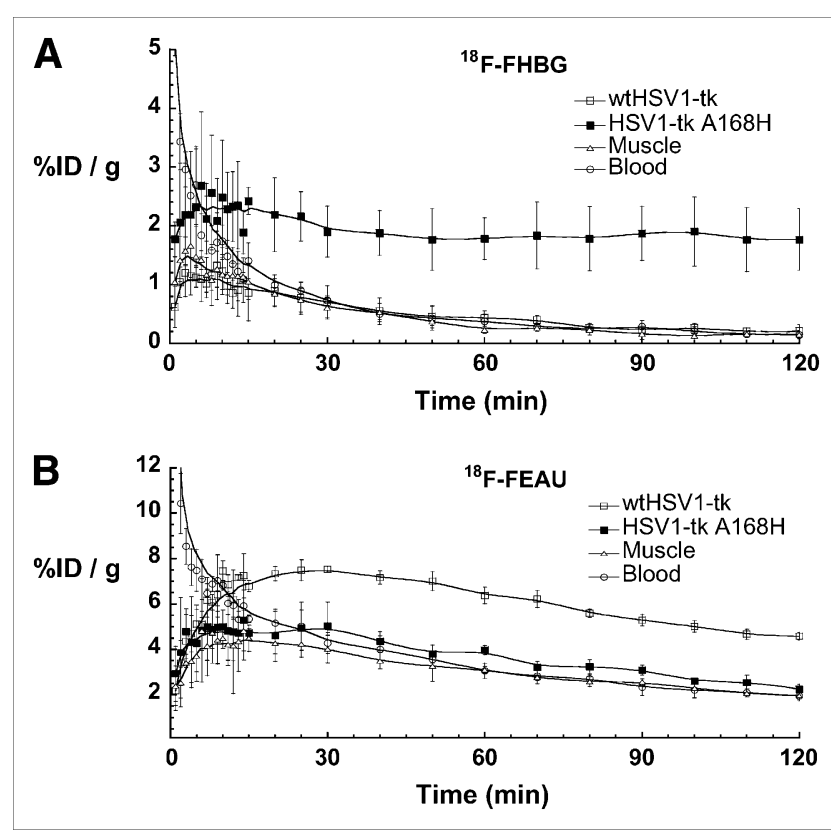

FIGURE 5. Time-activity curves indicate tumor-specific accumulation and differential uptake of ${ }^{18} \mathrm{~F}-\mathrm{FHBG}$ and ${ }^{18} \mathrm{~F}-$ FEAU. Analysis of ${ }^{18} \mathrm{~F}-\mathrm{FHBG}$ biodistribution in muscle, blood, and 2 U87 tumor types indicates time-resolved retention of radiotracer in U87 HSV1-tk(A168H)-expressing tumors (A). Although levels of ${ }^{18} \mathrm{~F}-\mathrm{FHBG}$ continued to decrease for duration of imaging session in U87 wtHSV1tk tumor, blood, and muscle, radiotracer levels in U87 HSV1$\mathrm{tk}(\mathrm{A} 168 \mathrm{H})$ mutant remained constant, leading to maximum resolution of image at $2 \mathrm{~h}$. Similar results were obtained for U87 wtHSV1-tk tumor with ${ }^{18} \mathrm{~F}-\mathrm{FEAU}(\mathrm{B})$.

\section{DISCUSSION}

In this article, we have shown that HSV1-tk(A168H), with its enhanced specificity for ${ }^{18} \mathrm{~F}-\mathrm{FHBG}$ and diminished catalytic activity for ${ }^{18} \mathrm{~F}-\mathrm{FEAU}$, should allow for differential imaging of 2 distinct molecular-genetic events by PET when used as a second reporter gene together with wtHSV1-tk. Our results also provide further support to the previously published report by Balzarini et al., which demonstrated that HSV1-tk(A168H), compared with wtHSV1-tk, possesses a 4-fold higher ganciclovir phosphorylation activity $\left(\mathrm{K}_{\mathrm{cat}}\right)$ and a 3- to 4-fold lower Thd phosphorylation activity (18).

The FHBG and FEAU binding affinity assessed here by computational analysis was consistent with observations made by Balzarini et al. (18) for the catalytic activity of wtHSV1-tk and the A168H mutant for Thd and acycloguanosine. Low Surflex docking scores indicated low pyrimidine substrate (Thd, FEAU, and FIAU) binding to the A168H mutant enzymes due to the clash of the ethyl, methyl, or iodine group at the $\mathrm{C} 5$ position of the uracil moiety of these substrates with $\mathrm{H} 168$ of the active site (Fig. 1). In contrast, the acycloguanosine substrates $\left({ }^{18} \mathrm{~F}-\mathrm{FHBG}\right.$, ganciclovir, and penciclovir) yielded higher Surflex scores with correct binding modes. Docking of FHBG into the active sites of the mutant enzymes is preserved because of a flexible planar shift of the purine ring away from the bulky side groups of H168. The accommodation of the acycloguanosine substrates into the mutant active sites may be attributed to the lack of an arabinoside moiety, providing conformational flexibility for the substrates in the spatially restrained active sites. This postulate is further substantiated by a recently published variant of HSV1-tk, HSV1-A167Ysr39tk, which contains bulkier active site substitutions A167Y and A168F. Like HSV1-tk(A168H), HSV1-A167Ysr39tk also exhibits diminished FEAU activity and enhanced FHBG phosphorylation activity over wtHSV1-tk (25).

The predicted outcome of the molecular modeling analysis was confirmed by the in vitro radiotracer accumulation studies. These results are reflected in the cellular uptake studies for the $\mathrm{A} 168 \mathrm{H}$ mutant. In the cells expressing HSV1-tk(A168H), no ${ }^{3} \mathrm{H}-\mathrm{FEAU}$ uptake was detected. Similarly, the ${ }^{14} \mathrm{C}$-Thd uptake rate was comparable to that in the untransduced U87 cells, indicating Thd phosphorylation by the endogenous thymidine kinase 1 without any detectable catalysis by the $\mathrm{A} 168 \mathrm{H}$ mutant. As predicted, the ${ }^{18} \mathrm{~F}-$ FHBG uptake rate was higher in the cells expressing HSV1-tk(A168H) than wtHSV1-tk, in agreement with previously reported results for ganciclovir (18).

Analysis of the HSV1-tk binding site provides insight into the enhanced acycloguanosine catalytic activity exhibited by the $\mathrm{A} 168 \mathrm{H}$ mutation. In evaluating the interaction of the A168H mutant with FHBG, we used the crystal structure of ganciclovir, with wtHSV1-tk as a basis for our discussion. In the crystal structure containing ganciclovir (1KI2), water 695 forms a hydrogen bond with the 2-amino group of ganciclovir in the vicinity of A168 and Y132 (26). In the minimized form of the $\mathrm{A} 168 \mathrm{H}$ mutant structure, the H168 side chain occupies the same 3-dimensional space in the binding site as does the water molecule (Fig. 6). This lends support to our suggestion that the histidine side chain would interact in a similar manner by hydrogen bonding to the equivalent amino group on FHBG, further stabilizing the substrate-enzyme complex and contributing to the enhanced catalytic activity.

Animal imaging results were consistent with in vitro uptake studies. U87 tumors expressing HSV1-tk(A186H) accumulated ${ }^{18} \mathrm{~F}$-FHBG more than an order of magnitude above blood and muscle levels and could be clearly distinguished from wtHSV-tk-expressing tumors, which exhibited only baseline (blood and muscle) levels of ${ }^{18} \mathrm{~F}-$ FHBG accumulation. Similarly, U87 wtHSV1-tk tumors were specifically and exclusively detectable with ${ }^{18} \mathrm{~F}-$ FEAU. Sufficient radiotracer accumulation was obtained to image and resolve the tumor from surrounding tissue, although the \%ID/g in U87 wtHSV1-tk tumors was only 2.3 -fold higher than the \%ID/g values of blood and muscle. U87 tumors expressing HSV1-tk(A168H) were not detectable with ${ }^{18} \mathrm{~F}-\mathrm{FEAU}$, yielding background levels of radiotracer uptake, as manifested by the low tumor-to-muscle and tumor-to-blood \% ID/g ratios of 1.13 and 1.12, respec- 


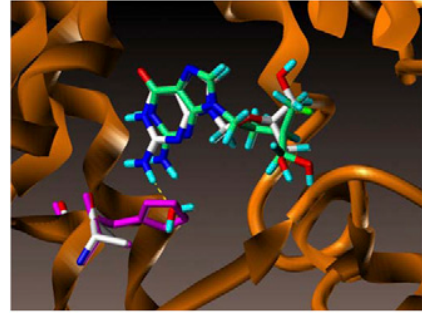

FIGURE 6. H168 occupies same 3 -dimensional space as water 695 in structure $1 \mathrm{KI}$. Formation of hydrogen bond between imidazole functional group of $\mathrm{H} 168$ (magenta) and 2-amino group of ${ }^{18} \mathrm{~F}-\mathrm{FHBG}$ (green structure) may plausibly account for enhanced acycloguanosine catalytic activity of mutant enzyme. In crystal structure 1KI2, water 695 forms hydrogen bond with ganciclovir substrate (26).

tively. The low tumor-to-muscle and tumor-to-blood ratios observed with ${ }^{18}$ F-FEAU in the U87 wtHSV1-tk tumors, compared with ${ }^{18} \mathrm{~F}-\mathrm{FHBG}$ accumulation in wtHSV1tk $(\mathrm{A} 168 \mathrm{H})$ tumors, may be attributed to a relatively slower systemic clearance of the ${ }^{18} \mathrm{~F}$-FEAU, which resulted in higher background levels of radioactivity in tissues at $2 \mathrm{~h}$ after injection.

The diminished catalytic activity for Thd exhibited by HSV1-tk(A168H) may enhance its utility as a PET reporter gene. Thus, its expression as a reporter may have less adverse influence on endogenous nucleotide metabolism (Thd-monophosphate pool) and, consequently, on the proliferative state of the cells. This feature was demonstrated by the in vitro uptake studies, in which ${ }^{14} \mathrm{C}$-Thd uptake in cells expressing HSV1-tk(A168H) was comparable to the levels observed in untransduced cells, in contrast to U87 cells expressing wtHSV1-tk.

The lack of Thd catalytic activity by HSV1-tk(A168H) also suggests that the reporter enzyme may be less influenced by endogenous levels of Thd. This results in an increase in ${ }^{18} \mathrm{~F}-\mathrm{FHBG}$ phosphorylation because of a lack of competition of this radiotracer with endogenous Thd levels. Furthermore, the rate of ${ }^{18} \mathrm{~F}-\mathrm{FHBG}$ accumulation in HSV1tk $(\mathrm{A} 168 \mathrm{H})$-expressing cells was 3-fold higher than wtHSV1-tk and twice as high as the rate of FEAU uptake by wtHSV1-tk. This phenomenon suggests that use of HSV1-tk(A168H) with ${ }^{18} \mathrm{~F}-\mathrm{FHBG}$ as a radiotracer may provide a more sensitive PET methodology than is currently available with wtHSV1-tk and ${ }^{18}$ F-FEAU. Comparison of PET technique sensitivity using HSV1-tk(A168H), wtHSV1-tk, and the HSV1-tk(SR39) variant (27) is currently in progress.

PET with ${ }^{18} \mathrm{~F}$-FHBG resulted in specific and distinct detection of the HSV1-tk(A168H)-expressing tumors, although wtHSV1-tk also possesses ${ }^{18}$ F-FHBG catalytic activity at low levels. The ${ }^{18} \mathrm{~F}-\mathrm{FHBG}$ accumulation rate in wtHSV1-tk-expressing U87 cells was, therefore, lower than in HSV1-tk(A168H)-expressing cells, and the uptake of ${ }^{18} \mathrm{~F}-\mathrm{FHBG}$ by wtHSV1-tk-expressing tumors was 4.9 -fold lower than the uptake rate of ${ }^{18} \mathrm{~F}$-FEAU. These differences in ${ }^{18} \mathrm{~F}-\mathrm{FHBG}$ accumulation rates between wtHSV1-tk and HSV1-tk(A168H) were adequate for achieving differential imaging of the 2 tumor types. Nonetheless, producing var- iants of HSV1-tk lacking catalytic activity for other purine or acycloguanosine-based radiotracers is desirable for more specific and sensitive differential imaging applications using pyrimidine-based radiotracers such as ${ }^{18}$ F-FEAU. Such reporter genes may be achievable by using mutant forms of thymidine kinase derived from ganciclovir-resistant strains of HSV1 $(28,29)$ that retain Thd phosphorylating activity.

\section{CONCLUSION}

We have demonstrated that effective differential PET of wtHSV1-tk and HSV1-tk(A168H) in tumors can be achieved using ${ }^{18} \mathrm{~F}-\mathrm{FEAU}$ and ${ }^{18} \mathrm{~F}-\mathrm{FHBG}$, respectively. This dual reporter gene-imaging approach can be further enhanced by creating a variant of HSV1-tk that lacks any detectable catalytic activity for ganciclovir, to completely eliminate any potential for signal overlap. The capacity to differentially image by PET will provide a valuable tool in many in vivo settings in which monitoring of 2 separate cellular events is required, such as the visualization of T-cells and their target tumor cell populations, monitoring of the trafficking of stem cells and their interaction with tumors, and simultaneous detection of viability and differentiation status of stem cells.

\section{REFERENCES}

1. Gelovani Tjuvajev J, Blasberg RG. In vivo imaging of molecular-genetic targets for cancer therapy. Cancer Cell. 2003;3:327-332.

2. Gambhir SS. Molecular imaging of cancer with positron emission tomography. Nat Rev Cancer. 2002;2:683-693.

3. Blasberg RG, Gelovani J. Molecular-genetic imaging: a nuclear medicine-based perspective. Mol Imaging. 2002;1:280-300.

4. Tjuvajev JG, Finn R, Watanabe K, et al. Noninvasive imaging of herpes virus thymidine kinase gene transfer and expression: a potential method for monitoring clinical gene therapy. Cancer Res. 1996;56:4087-4095.

5. Tjuvajev JG, Avril N, Oku T, et al. Imaging herpes virus thymidine kinase gene transfer and expression by positron emission tomography. Cancer Res. 1998;58:4333-4341.

6. Serganova I, Doubrovin M, Vider J, et al. Molecular imaging of temporal dynamics and spatial heterogeneity of hypoxia-inducible factor-1 signal transduction activity in tumors in living mice. Cancer Res. 2004;64:6101-6108.

7. Hajitou A, Trepel M, Lilley CE, et al. A hybrid vector for ligand-directed tumor targeting and molecular imaging. Cell. 2006;125:385-398.

8. Soghomonyan S, Hajitou A, Rangel R, et al. Molecular PET imaging of HSVl-tk reporter gene expression using $\left[{ }^{18}\right.$ F]FEAU. Nat Protocols. 2007;2:416-423.

9. Alauddin MM, Conti PS. Synthesis and preliminary evaluation of $9-\left(4-\left[{ }^{18} \mathrm{~F}\right]-\right.$ fluoro-3-hydroxymethylbutyl)guanine ([ $\left.\left.{ }^{18} \mathrm{~F}\right] \mathrm{FHBG}\right)$ : a new potential imaging agent for viral infection and gene therapy using PET. Nucl Med Biol. 1998; 25:175-180.

10. Yaghoubi S, Barrio JR, Dahlbom M, et al. Human pharmacokinetic and dosimetry studies of $\left[{ }^{18} \mathrm{~F}\right] \mathrm{FHBG}$ : a reporter probe for imaging herpes simplex virus type-1 thymidine kinase reporter gene expression. J Nucl Med. 2001; 42:1225-1234

11. Yaghoubi SS, Gambhir SS. PET imaging of herpes simplex virus type 1 thymidine kinase (HSV1-tk) or mutant $H S V 1$-sr39tk reporter gene expression in mice and humans using $\left[{ }^{18}\right.$ F]FHBG. Nat Protocols. 2007;1:3069-3074.

12. Penuelas I, Haberkorn U, Yaghoubi S, Gambhir SS. Gene therapy imaging in patients for oncological applications. Eur J Nucl Med Mol Imaging. 2005; 32(suppl 2):S384-S403.

13. Jacobs A, Voges J, Reszka R, et al. Positron-emission tomography of vectormediated gene expression in gene therapy for gliomas. Lancet. 2001;358:727-729.

14. Kang KW, Min JJ, Chen X, Gambhir SS. Comparison of $\left[{ }^{14} \mathrm{C}\right] \mathrm{FMAU}$, $\left[{ }^{3} \mathrm{H}\right] \mathrm{FEAU},\left[{ }^{14} \mathrm{C}\right] \mathrm{FIAU}$, and $\left[{ }^{3} \mathrm{H}\right] \mathrm{PCV}$ for monitoring reporter gene expression of wild type and mutant herpes simplex virus type 1 thymidine kinase in cell culture. Mol Imaging Biol. 2005;7:296-303. 
15. Ponomarev V, Doubrovin M, Shavrin A, et al. A human-derived reporter gene for noninvasive imaging in humans: mitochondrial thymidine kinase type 2 . J Nucl Med. 2007;48:819-826.

16. Kummer C, Winkeler A, Dittmar C, et al. Multitracer positron emission tomographic imaging of exogenous gene expression mediated by a universal herpes simplex virus 1 amplicon vector. Mol Imaging. 2007;6:181-192.

17. Shin JH, Chung JK, Kang JH, et al. Feasibility of sodium/iodide symporter gene as a new imaging reporter gene: comparison with HSV1-tk. Eur J Nucl Med Mol Imaging. 2004;31:425-432.

18. Balzarini J, Liekens S, Solaroli N, El Omari K, Stammers DK, Karlsson A. Engineering of a single conserved amino acid residue of herpes simplex virus type 1 thymidine kinase allows a predominant shift from pyrimidine to purine nucleoside phosphorylation. J Biol Chem. 2006;281:19273-19279.

19. Berman HM, Westbrook J, Feng Z, et al. The protein data bank. Nucleic Acids Res. 2000;28:235-242.

20. Case DA, Cheatham TE III, Darden T, et al. The Amber biomolecular simulation programs. J Comput Chem. 2005;26:1668-1688.

21. Jain AN. Surflex: fully automatic flexible molecular docking using a molecular similarity-based search engine. J Med Chem. 2003;46:499-511.

22. Jain AN. Ligand-based structural hypotheses for virtual screening. J Med Chem. 2004;47:947-961.
23. Ponomarev V, Doubrovin M, Serganova I, et al. Cytoplasmically retargeted HSV1-tk/GFP reporter gene mutants for optimization of noninvasive moleculargenetic imaging. Neoplasia. 2003;5:245-254.

24. Ory DS, Neugeboren BA, Mulligan RC. A stable human-derived packaging cell line for production of high titer retrovirus/vesicular stomatitis virus $G$ pseudotypes. Proc Natl Acad Sci USA. 1996;93:11400-11406.

25. Likar Y, Dobrenkov K, Olszewska M, et al. A new acycloguanosine-specific supermutant of herpes simplex virus type 1 thymidine kinase suitable for PET imaging and suicide gene therapy for potential use in patients treated with pyrimidine-based cytotoxic drugs. J Nucl Med. 2008;49:713-720.

26. Champness JN, Bennett MS, Wien F, et al. Exploring the active site of herpes simplex virus type-1 thymidine kinase by X-ray crystallography of complexes with aciclovir and other ligands. Proteins. 1998;32:350-361.

27. Gambhir SS, Bauer E, Black ME, et al. A mutant herpes simplex virus type 1 thymidine kinase reporter gene shows improved sensitivity for imaging reporter gene expression with positron emission tomography. Proc Natl Acad Sci USA. 2000;97:2785-2790.

28. Gilbert C, Bestman-Smith J, Boivin G. Resistance of herpesviruses to antiviral drugs: clinical impacts and molecular mechanisms. Drug Resist Updat. 2002;5:88-114.

29. Morfin F, Thouvenot D. Herpes simplex virus resistance to antiviral drugs. J Clin Virol. 2003;26:29-37. 